\title{
Bioinformatic analysis of retinal gene function and expression in diabetic rats
}

\author{
WENJUAN ZHAO ${ }^{1}$, DONG WANG $^{2}$, JUN ZHAO $^{3}$ and WENQING ZHAO ${ }^{4}$ \\ ${ }^{1}$ Department of Ophthalmology, Shandong University Affiliated Jinan Central Hospital, Jinan, Shandong 250013; \\ ${ }^{2}$ School of Management Science and Engineering, Shandong University of Finance and Economics, Jinan, \\ Shandong 250014; ${ }^{3}$ Health Examination Center, The Second People's Hospital of Jinan, Jinan, Shandong 250001; \\ ${ }^{4}$ Department of Neurosurgery, The Fifth People's Hospital of Jinan, Jinan, Shandong 250022, P.R. China
}

Received May 9, 2016; Accepted April 7, 2017

DOI: $10.3892 /$ etm.2017.4805

\begin{abstract}
The aim of the present study was to investigate the changes in retinal gene expression at three time points and assess the underlying molecular mechanisms of diabetic retinopathy (DR) in a streptozotocin (STZ)-induced diabetes rat model using bioinformatics analysis. The gene expression profile of GSE28831 was extracted from the Gene Expression Omnibus database and differentially expressed genes (DEGs) were identified at three different time points (1,4 and 12 weeks) using the limma package in R language. Gene ontology (GO) enrichment analysis of DEGs was performed followed by a principal component and pathway enrichment analysis of the selected DEGs along with protein-protein interaction network construction at the three time points. A total of 402, 105 and 213 DEGs were screened at 1, 4 and 12 weeks, respectively. In addition, the expression of 8 genes was identified to be significantly different at different time points, including cytochrome P450 2B2 (CYP2B2; downregulated gene; $\mathrm{P}=0.048$; at 1 week), mannan binding lectin-associated serine protease-2 (MASP2; downregulated gene; $\mathrm{P}=0.044)$, lecithin retinol acyltransferase (LRAT; downregulated gene; $\mathrm{P}=0.015$ ), retinal pigment epithelium (RPE)-specific protein $65 \mathrm{kDa}$ (RPE65; downregulated gene; $\mathrm{P}=0.025$ ), 11-cis-retinoldehydrogenase (RDH5; downregulated gene; $\mathrm{P}=0.04$; at 4 weeks), mitogen-activated protein kinase 13 (MAPK13; upregulated gene; $\mathrm{P}=0.036$ ), LRAT (downregulated gene; $\mathrm{P}=0.01$ ) and RPE65 (downregulated gene; $\mathrm{P}=0.009$; at 12 weeks). Furthermore, pathway enrichment and GO enrichment analyses revealed that DEGs at 4 weeks were primarily enriched in retinol metabolism and processes associated with visual functions, including 'visual perception'
\end{abstract}

Correspondence to: Dr Wenqing Zhao, Department of Neurosurgery, The Fifth People's Hospital of Jinan, 24297 Jingshi Road, Jinan, Shandong 250022, P.R. China

E-mail: 1iinhai@163.com

Key words: diabetic retinopathy, differentially expressed gene, principal component analysis, pathway enrichment analysis, function enrichment analysis and 'retinol metabolism'. DEGs, including CYP2B2, MASP2, LRAT, RPE65, RDH5 and MAPK13 may be potential targets for the diagnosis and treatment of DR. Thus, the current study demonstrated that abnormal visual functions occur at 4 weeks in STZ-induced diabetic rats. This may provide a scientific basis for the diagnosis and treatment of DR because DEGs may be used to facilitate the development of novel therapeutic strategies to diagnose and treat DR.

\section{Introduction}

Diabetic retinopathy (DR) is a microvascular complication and is the most common cause of vision impairment and blindness among adults aged 20-74 years (1). The incidence of DR is associated with a number of risk factors such as hyperglycemia, hyperlipidemia and hypertension (2) and is characterized by signs of retinal ischemia and/or signs of increased retinal vascular permeability (3). Furthermore, DR is a progressive disease that endangers all retinal layers via insults of metabolic and neurological inflammation, which may contribute to vascular disruptions over time (4). Although clinical trials have demonstrated that effective treatments for diabetic retinopathy may reduce severe vision loss by $90 \%$ (5-7), these studies have emphasized the critical requirement for periodic eye examinations for all patients with diabetes. Thus, it is necessary to improve the means by which retinopathy is identified and prevented in its earliest stages rather than wait for the onset of vision-threatening lesions (8).

Over the past decade, studies have demonstrated that numerous factors may be involved in the pathogenesis of DR $(9,10)$ and it has been determined that chronic hyperglycemia is a factor involved in the development and progression of DR (11-13). Chronic hyperglycemia may induce multiple cellular changes that lead to diabetic complications due to its toxic effects and/or the effects of its pathophysiological derivatives that directly act on tissues $(14,15)$. Other factors including non-enzymatic glycation and glycoxidation may also induce DR. Furthermore, a number of candidate genes associated with the visual cycle, including lecithin retinol acyltransferase (LRAT) (16), retinal pigment epithelium (RPE)-specific protein $65 \mathrm{kDa}$ (RPE65) (17) and 11-cis-retinoldehydrogenase (RDH5) (18) have been implicated in DR. Although early 
changes in retinal gene expression have been evaluated by analyzing retinal gene expression in streptozotocin (STZ)-induced diabetic rats (19), the specific underlying molecular mechanisms that occur during DR development and progression remain to be elucidated.

In the present study, bioinformatics was used to analyze the changes in retinal gene expression over a period of 12 weeks. Differentially expressed genes were analyzed at three different time points (1, 4 and 12 weeks) in the gene expression profile of retinal samples. The present study assessed the changes in retinal gene expression at the three time points with the aim of identifying the underlying molecular mechanisms of DR in diabetes.

\section{Materials and methods}

Affymetrix microarray data of retina gene. The gene expression profile of GSE28831 was downloaded from the Gene Expression Omnibus database (https://www .ncbi.nlm.nih.gov/geo/query/acc.cgi?acc=GSE28831). The dataset of GSE28831 was provided by a previous study by Kirwin et al (19), which obtained retinal samples of Long Evans rats, sacrificed 7 days, 4 weeks and 3 months after the induction of diabetes. A total of 21 retinal samples including 12 control samples and 9 diabetic samples were eligible for further analysis. Control samples consisted of 9 normal samples and 3 samples that received an injection of streptozotocin (STZ), but did not develop hyperglycemia. Diabetes samples included 9 retina samples which were obtained from STZ-induced diabetic rats. Retinal samples were collected from the retinas of control $(n=3)$ and STZ-induced diabetic rats $(n=3)$ at the three time points $(1$, 4 and 12 weeks after the induction of diabetes). In addition, 3 other samples were obtained from rats that were normoglycemic 1 week following STZ treatment that were used as normal controls.

The platform used was the GPL7294 Agilent-014879 Whole Rat Genome Microarray 4x 44K G4131F (Agilent Technologies, Inc., Santa Clara, CA, USA). The raw file and the annotation information file of the platform were also downloaded.

Data preprocessing and differential expression analysis. Each probe was mapped with one or multiple gene names labeled in an annotation platform. To make statistical inferences, each gene was normalized between microarrays based on quartile normalization (20). When multiple probes were mapped to the same gene ID, the mean expression of those probes was calculated. When one probe was mapped to multiple gene sets, information about the probe was deleted. The linear models of microarray data (limma) package (Version 3.30.3; Bioconductor, Seattle, WI, USA) in R language was used to identify genes that were differentially expressed between control and disease samples at the three time points (21). The $\mathrm{P}$ values of genes were calculated using student's t test and adjusted by multiple testing to circumvent the false-positive results. Subsequently, the $\log 2$-fold-change $(\log \mathrm{FC})$ was calculated. Only genes with an adjusted $\mathrm{P}<0.05$ and $\mid \log _{2} \mathrm{FCl}>1$ were selected as differentially expressed genes (DEGs) (22), which were the signature genes of DR.
Comparison of DEGs between control and disease samples. The DEGs between control and disease samples at the three time points were classified into upregulated and downregulated gene sets for further analysis. Subsequently, the contrast between the DEGs at the three time points was assessed according to the number of DEGs. Then gene sets at the three time points were compared and illustrated using a Venn diagram (Fig. 1) (23).

Principal component analysis (PCA) of DEGs. PCA, a multivariate regression analysis (24), was used to distinguish samples with multiple measurements (25). A PCA of DEGs was conducted in the present study using prcomp algorithm in $\mathrm{R}$ language $(26,27)$. A 3D graph was then obtained, in which DEGs were considered as variables and the difference between control and disease samples at the three time points were observed.

Pathway enrichment analysis of DEGs. Pathway enrichment analysis is widely used to analyze high-throughput data and help link individual genes or proteins, which are differentially expressed under specific conditions, in order to improve understanding regarding biological pathways (28). To identify the pathways associated with DEGs selected at the three time points, the software tool KOBAS [kyoto encyclopedia of genes and genomes (KEGG) Orthology Based Annotation System] version 2 (http://kobas.cbi.pku.edu.cn), which was used for the annotation and identification of enriched pathways and diseases (29) based on the cumulative hypergeometric distribution algorithm (a discrete probability distribution), which was used for drawing from $>2$ selections in a population, again without replacement (30) with $\mathrm{P}<0.05$ denoting a significant difference.

Protein-Protein interaction (PPI) network construction. Numerous physical activities of the cells were evaluated based on protein integration and dissociation. A variety of cellular physiological activities and the reaction of cells to the external and internal environment are regulated by PPI networks (31). Thus, a more thorough investigation of protein interactions is required to understand this biological phenomenon (32).

STRING (search tool for retrieving interacting genes/proteins; http://string-db.org/) (33) provides uniquely comprehensive coverage and ease of access to experimental and predicted interaction information. A PPI network was constructed, in which the interactions of DEGs between control and disease samples at the three time points were mapped to STRING based on information including the sequence characters and structures. Interaction patterns with a combined score $>0.4$ were selected. Subsequently, the PPI network at the three time points was visualized using Cytoscape software (version 3.4.0; Cytoscape Consortium, San Diego, CA, USA) (34). The nodes with higher degree served an important role in the PPI network, and may be the key nodes. Nodes represent the proteins encoded by DEGs, and a degree is considered to be the numbers of proteins that interact with that protein.

Functional analysis of the DEGs at the three time points. Gene ontology enrichment analysis has been extensively 
A

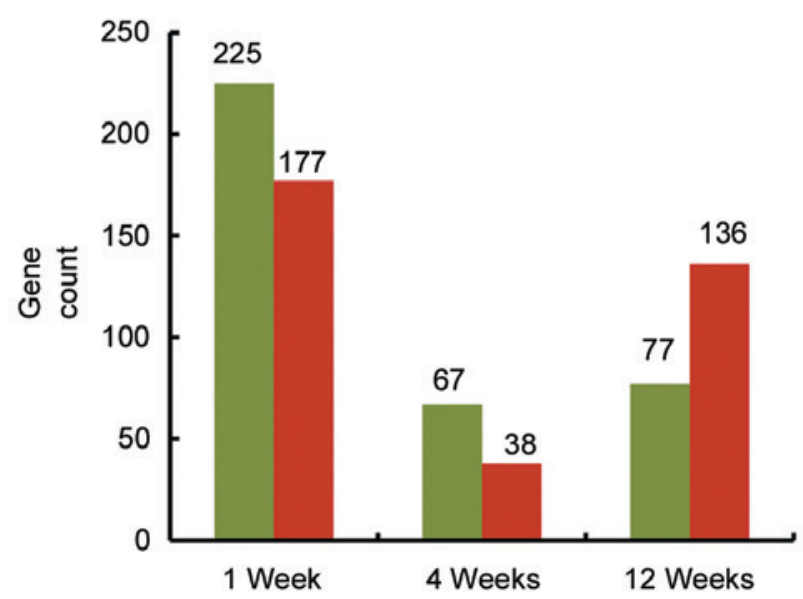

B

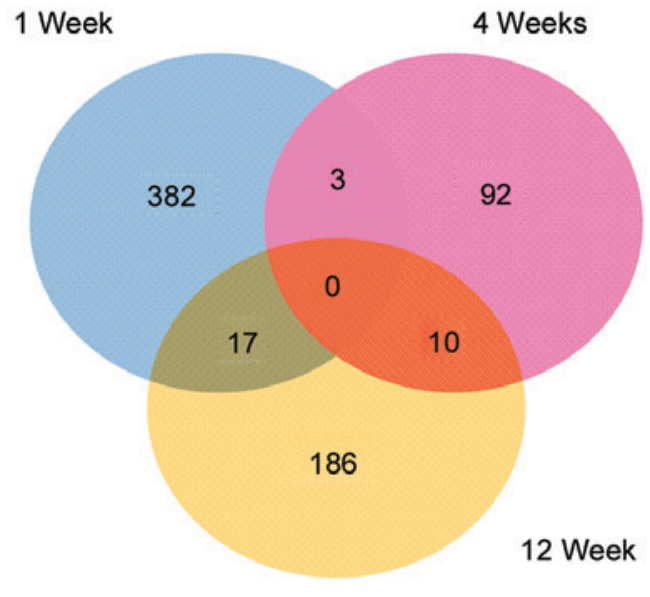

Figure 1. Comparision of differentially expressed genes at the three timepoints. (A) Historgram graph demonstrating the number of upregulated and downregulated genes at three time points. Green represents upregulated genes and red represents downregulated genes. (B) Venn diagram of the number of genes at each timepoint. Blue represents 1 week, pink represents 4 weeks and yellow represents 12 weeks.

A

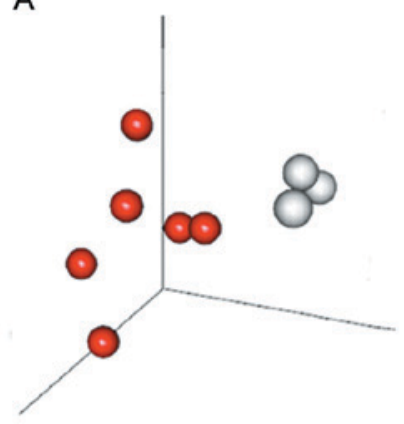

B

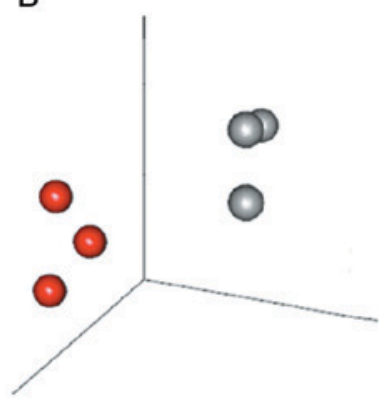

C

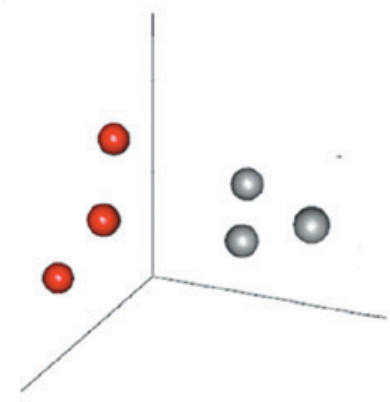

Figure 2. Principal component analysis of DEGs at the three timepoints. Analysis of DEGs at (A) 1 , (B) 4 and (C) 12 weeks. Red pellets, STZ-induced rat retinal sample; gray pellets, normal rat retinal sample; DEGs, differentially expressed genes.

used to provide a detailed view for understanding the molecular mechanism and identifying a deeply complicated network, which consisted of a set of genes with similar functions (35).

DAVID (a database for annotation, visualization, and integrated discovery; version 6.8; https://david.ncifcrf.gov/home. jsp) (36), a comprehensive set of functional annotation tools, was used for systematic and integrative analyses of large gene/protein lists. It evaluated the functions of the DEGs at the three time points and defined significant function enrichment of these genes in multiple gene ontology (GO) categories when $\mathrm{P}<0.05$.

\section{Results}

Identification of DEGs. A total of 402, 105 and 213 DEGs with the cut-off criteria of an adjusted value of significance $(\mathrm{P}<0.05)$ and $\log _{2} \mathrm{FCl}>1$ were selected as the thresholds to screen DEGs in control and disease samples at 1,4 and 12 weeks.

Comparison of DEGs at the three time points. As presented in Fig. 1A, a total of 720 DEGs including 369 upregulated and 351 downregulated DEGs were screened. There were a greater number of downregulated than upregulated DEGs, with the exception of at 12 weeks following STZ-induced diabetes where the converse was true.

As presented in Fig. 1B, there were no common DEGs at all three time points. However, there were 3 common DEGs in weeks 1 and 4, 17 common DEGs in weeks 1 and 12 and 10 common DEGs in weeks 4 and 12.

PCA of DEGs. As presented in Fig. 2, disease and control samples were completely separated by the selected DEGs at the three time points, meaning that the expression patterns of screened DEGs were specific at different time points and could be used to completely distiguish between disease and control samples.

Pathway enrichment analysis of the DEGs. The significantly enriched pathways for the up- and downregulated DEGs are presented in Table I. There were two KEGG pathways (Neuroactive ligand-receptor interaction and Arachidonic acid metabolism) associated with DEGs at 1 week and one KEGG pathway (Retinol metabolism) associated with DEGs at 4 weeks. The retinol metabolism pathway included three downregulated genes (LRAT, RPE65 and RDH5) and the 
Table I. DEGs enriched in the KEGG pathway at the three time points.

A, Week 1

\begin{tabular}{lcl}
\hline Term & Count & \multicolumn{1}{c}{ Genes } \\
\hline $\begin{array}{l}\text { rno04080: Neuroactive ligand-receptor } \\
\text { interaction }\end{array}$ & 13 & THRB, LEPR, VIPR1, \\
& & NPY5R, CHRM5, GPR35, \\
& & CHRM4, PTGDR, ADRA1B, \\
& & MTNR1B, ADRA2B, \\
rno00590: Arachidonic acid metabolism & 5 & HTR2A, GHR \\
& & CYP2U1, CYP2B2, PLA2G4A, \\
& & CYP4F17, GPX3 \\
\hline
\end{tabular}

B, Week 4

\begin{tabular}{lccc}
\hline Term & Count & Genes & P-value \\
\hline rno00830: Retinol metabolism & 3 & LRAT, RPE65, RDH5 & 0.041873 \\
\hline
\end{tabular}

DEGs, differentially expressed genes; KEGG, kyoto encyclopedia of genes and genomes.
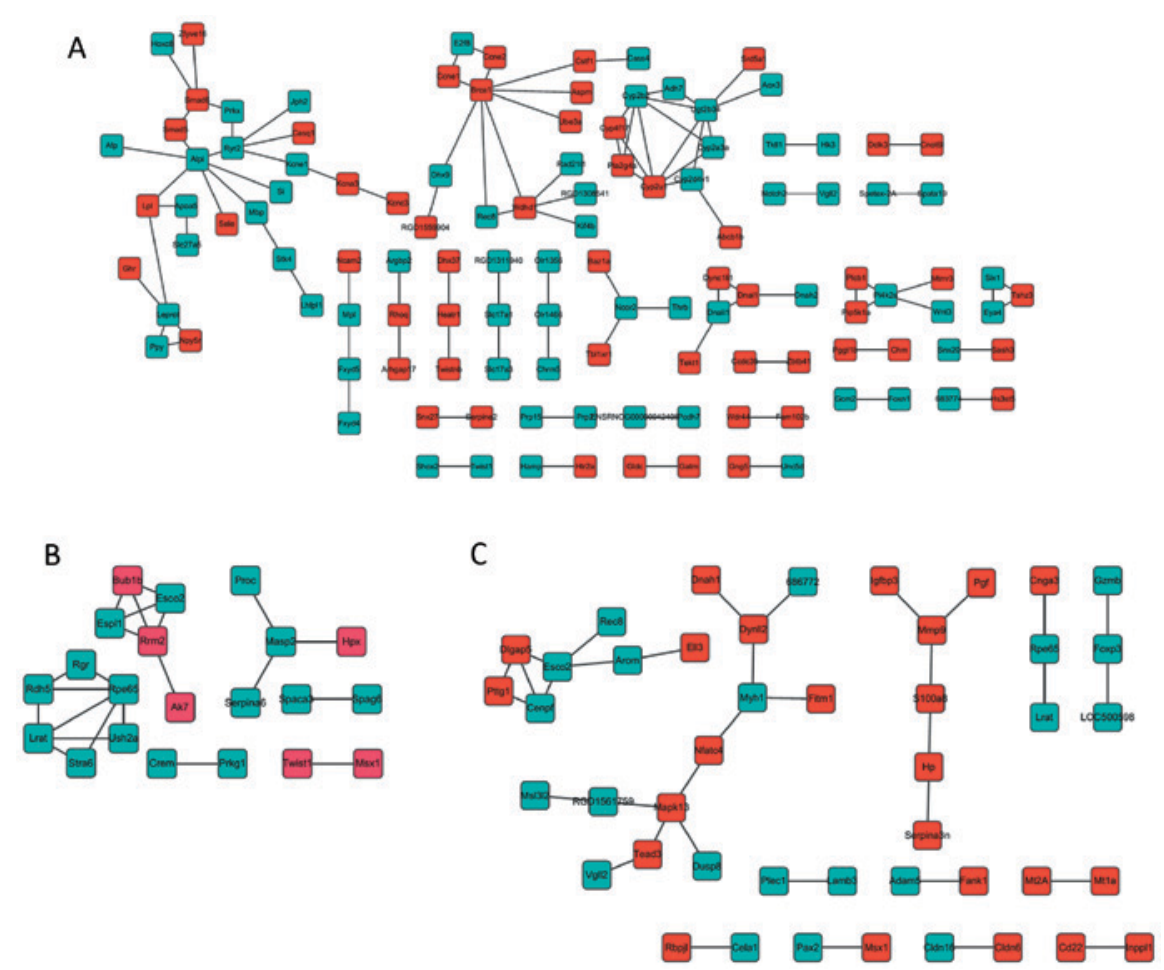

Figure 3. Protein-protein interaction network at the three timepoints. Network at (A) 1, (B) 4 and (C) 12 weeks. Green nodes represent proteins expressed by downregulated differentially expressed genes; red nodes represent proteins expressed by upregulated differentially expressed genes.

LRAT and RPE65 genes were also downregulated in DEGs at 12 weeks (Fig. 3).

PPInetwork construction. A total of 104, 22 and 35 PPI pairs at 1, 4 and 12 weeks, respectively were obtained. These PPI pairs were then used to construct the PPI network by integrating these associations in the retina (Fig. 3). In this network, the proteins cytochrome P450 2B2 (CYP2B2; 1 week), mannan binding lectin-associated serine protease-2 (MASP2), LRAT,
RPE65, RDH5 (4 weeks), mitogen-activated protein kinase 13 (MAPK13), LRAT and RPE65 (12 weeks) with high degrees form a local network and these proteins served an important role in the PPI network, and may be the key proteins in the development of DR.

GO enrichment analysis of DEGs involved in PPI network. As presented in Table II, the DEGs in the PPI network at 1, 4 and 12 weeks were enriched in 12,7 and 10 GO categories, 


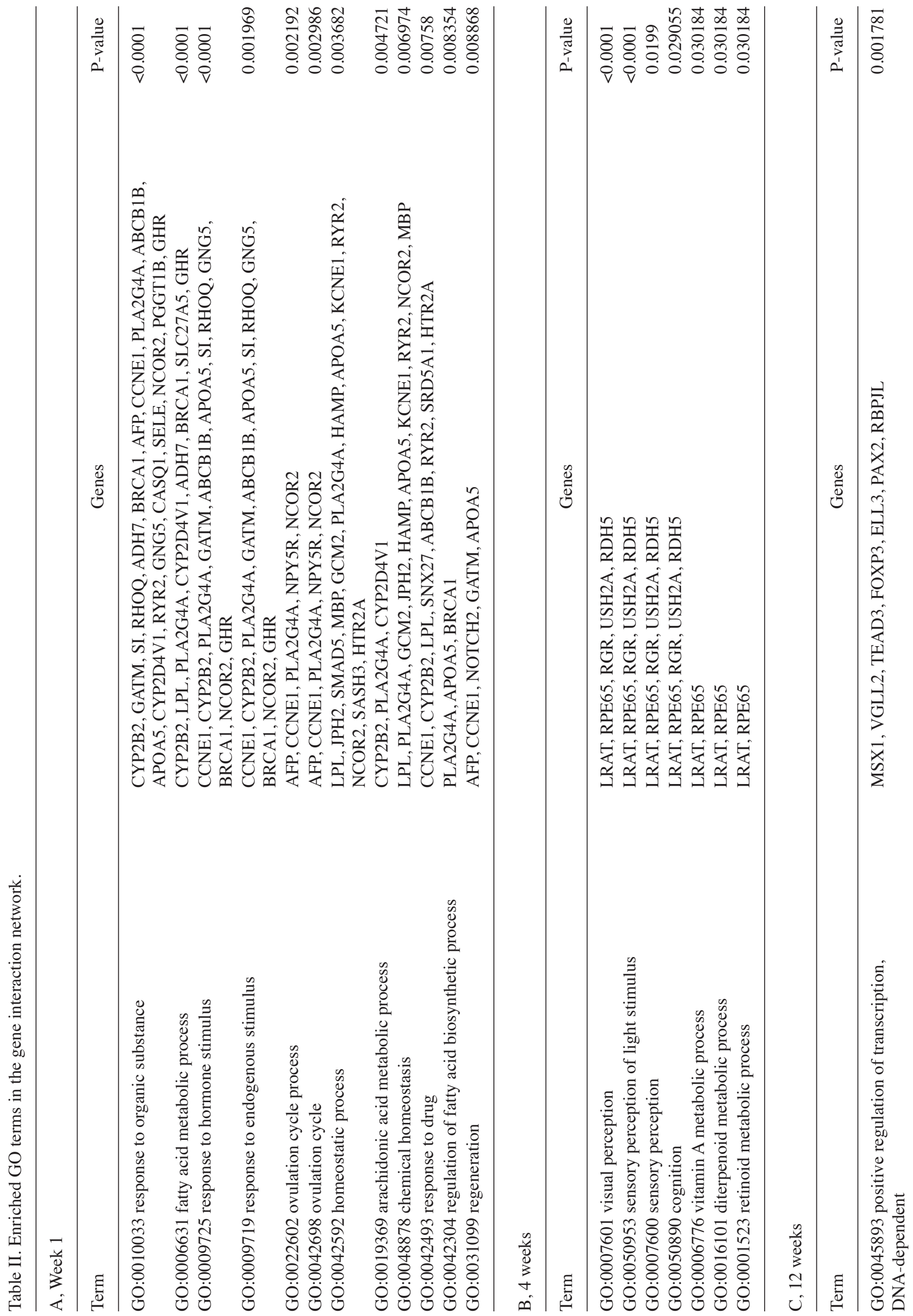


nิ $\mid$ respectively. Furthermore the DEGs in PPI network at 1 week were primarily enriched in processes associated with metabolism and response, including 'fatty acid metabolic processes', 'responses to drug' and 'responses to endogenous stimulus'. The DEGs in the PPI network at 4 weeks were enriched in processes associated with visual functions, such as 'visual perception', 'sensory perception of light stimulus' and 'vitamin A metabolic process'. The DEGs in the PPI network at 12 weeks were primarily enriched in regulatory processes including 'positive regulation of transcription, DNA-dependent', 'positive regulation of RNA metabolic process' and 'negative regulation of cell proliferation'.

\section{Discussion}

Due to the increasing number of individuals diagnosed with diabetes, blindness caused by DR may become more common unless improvements are made in the diagnosis and treatment of DR (7). To improve understanding regarding the mechanisms involved in DR formation, the present study investigated the biological processes and signaling pathways associated with DR. The analysis of gene expression profiling identified the abnormally expressed genes associated with DR and enabled the identification of novel targets for therapeutic strategies. In the current study, a total of 402, 105 and 213 DEGs were screened at 1,4 and 12 weeks, respectively, and eight significant genes were identified including CYP2B2 (at 1 week), MASP2, LRAT, RPE65, RDH5 (at 4 weeks), MAPK13, LRAT and RPE65 (at 12 weeks). Pathway enrichment and GO enrichment analyses revealed that DEGs were primarily enriched in processes associated with visual functions, including 'visual perception' and 'retinol metabolism' at 4 weeks.

CYP2B2 encodes an enzyme that controls arachidonic acid metabolism (37). Arachidonic acid is a precursor of inflammatory mediators, and early stage DR is regarded as a low-grade chronic inflammatory condition (38). Pathway enrichment analysis in the present study revealed that DEGs at 1 week were enriched in arachidonic acid metabolism. However, early inflammation of the eyes does not lead to any clinical manifestations. Therefore, inflammation in the retina may be an important component of early DR without clinical manifestations and the associated genes, including CYP2B2, may serve important roles in the evaluation of these functions. This is in accordance with a previous study by Joussen et al (39), which suggested that the response of the retina to the diabetic challenge consists of an inflammatory component.

In the present study, MASP2 was downregulated at 4 weeks. MASP2 encodes a novel serine protease that acts in the mannan-binding lectin (MBL)-lectin complement fixation pathway (40). The MBL-lectin complement fixation pathway is widely thought to serve a major role in host defense against infection and the expression of MBL is upregulated during inflammation (41). Østergaard et al (42) suggested that the complement system, specifically the MBL pathway, serves an important role in the pathogenesis of diabetic vascular complications such as DR. The decreased expression of MASP2 leads to MBL deficiency, which increases the overall susceptibility of an individual to infectious diseases (43). Thus, the present study suggests that the downregulation of MASP2 may 
stimulate the progression of DR and promote the appearance of other symptoms associated with DR.

LRAT, RPE65 and RDH5 are involved in retinol metabolism, and these genes are all part of the visual cycle that recycles all-trans-retinal from bleached photoreceptors to the 11-cis-retina required for phototransduction (20). The visual cycle is critical for visual function. Previous studies have reported that mutations in three different genes involved in the visual cycle, RPE65, LRAT and RDH12 cause early onset retinal dystrophy (44). Furthermore, RPE65 is essential for the maintenance of normal vision $(45,46)$. Thus, the downregulation of LRAT, RPE65 and RDH5 may lead to abnormal visual function. In addition, LRAT and RPE65 were included in the downregulated DEGs at 12 weeks and GO enrichment analysis revealed that he majority of DEGs at 4 weeks were associated with visual functions, including 'visual perception', 'sensory perception of light stimulus' and 'vitamin A metabolic process'. It has therefore been suggested that abnormal visual functions occur at 4 weeks in STZ-induced diabetic rats, which may be caused by the downregulation of genes associated with visual functions, including LRAT, RPE65 and RDH5. However, considering the present study is a bioinformatic study, further long-term studies are required to verify the association between gene expression and visual function.

MAPK13 encodes an enzyme that is included in the MAPK family. In the PPI network at 12 weeks, MAPK13 with a high degree formed a local network, suggesting that MAPK13 may serve a major role in DR. MAPKs have been implicated in a number of diseases including cancer, inflammatory disease, obesity and diabetes $(47,48)$. Previous studies have reported that MAPKs are involved in regulating insulin secretion and $\beta$ cell survival $(47,49,50)$ and MAPK inhibitors contribute to the prevention of cardiovascular diseases such as retinopathy (51). Therefore, MAPK13 may be involved in the pathogenesis of DR, although further studies are required to confirm this observation.

In conclusion, the data in the present study provide comprehensive bioinformatic analysis of DEGs, which may be involved in DR. It was also demonstrated that abnormalities in visual functions occur in STZ-induced diabetic rats at 4 weeks. DEGs, including CYP2B2, MASP2, LRAT, RPE65, RDH5 and MAPK13 may be potential targets for DR diagnosis and treatment. The results of the current study may provide a scientific basis for the diagnosis and targeted therapy of DR.

\section{References}

1. Fong DS, Aiello L, Gardner TW, King GL, Blankenship G, Cavallerano JD, Ferris FL III and Klein R; American Diabetes Association: Retinopathy in diabetes. Diabetes Care 27 (Suppl 1): S84-S87, 2004.

2. Malone JI, Morrison AD, Pavan PR and Cuthbertson DD; Diabetic Control and Complications Trial: Prevalence and significance of retinopathy in subjects with type 1 diabetes of less than 5 years' duration screened for the diabetes control and complications trial. Diabetes Care 24: 522-526, 2001.

3. Kempen JH, O'Colmain BJ, Leske MC, Haffner SM, Klein R, Moss SE, Taylor HR and Hamman RF; Eye Diseases Prevalence Research Group: The prevalence of diabetic retinopathy among adults in the united states. Arch Ophthalmol 122: 552-563, 2004

4. Ciulla TA, Amador AG and Zinman B: Diabetic retinopathy and diabetic macular edema: Pathophysiology, screening, and novel therapies. Diabetes Care 26: 2653-2664, 2003.
5. Photocoagulation treatment of proliferative diabetic retinopathy: The second report of diabetic retinopathy study findings. Ophthalmology 85: 82-106, 1978.

6. Early photocoagulation for diabetic retinopathy. ETDRS report number 9 . Early treatment diabetic retinopathy study research group. Ophthalmology 98 (5 Suppl): S766-S785, 1991.

7. Wilkinson C, Ferris FL III, Klein RE, Lee PP, Agardh CD, Davis M, Dills D, Kampik A, Pararajasegaram R and Verdaguer JT; Global Diabetic Retinopathy Project Group: Proposed international clinical diabetic retinopathy and diabetic macular edema disease severity scales. Ophthalmology 110: 1677-1682, 2003.

8. Antonetti DA, Barber AJ, Bronson SK, Freeman WM, Gardner TW, Jefferson LS, Kester M, Kimball SR, Krady JK, LaNoue KF, et al: Diabetic retinopathy: seeing beyond glucose-induced microvascular disease. Diabetes 55: 2401-2411, 2006.

9. Sulochana KN, Ramakrishnan S, Rajesh M, Coral K and Badrinath SS: Diabetic retinopathy: Molecular mechanisms, present regime of treatment and future perspectives. Curr Sci 80: 133-142, 2001.

10. Takagi H, Oh H, Otani A, Suzuma K, Suzuma I, Ohashi H, Wantanabe D, Kemmochi S, Ojima T, Suganami E, et al: Molecular mechanisms of retinal neovascularization in diabetic retinopathy. Int Congress Ser 1262: 160-163, 2004.

11. Klein R, Klein BK, Moss SE and Cruickshanks KJ: Relationship of hyperglycemia to the long-term incidence and progression of diabetic retinopathy. Arch Intern Med 154: 2169-2178, 1994.

12. Engerman RL and Kern TS: Hyperglycemia as a cause of diabetic retinopathy. Metabolism 35 (4 Suppl 1): S20-S23, 1986.

13. Balasubramanyam M, Rema M and Premanand C: Biochemical and molecular mechanisms of diabetic retinopathy. Curr Sci 83: 1506-1514, 2002.

14. Sheetz MJ and King GL: Molecular understanding of hyperglycemia's adverse effects for diabetic complications. JAMA 288: 2579-2588, 2002.

15. Nishikawa T, Edelstein D and Brownlee M: The missing link: A single unifying mechanism for diabetic complications. Kidney Int Suppl 77: S26-S30, 2000

16. Larsen LM: Prevention of retinopathy by inhibition of the visual cycle. US Patent 9421175 B2. Filed March 16, 2005; issued August 23, 2016.

17. Jin M, Li S, Moghrabi WN, Sun H and Travis GH: Rpe65 is the retinoid isomerase in bovine retinal pigment epithelium. Cell 122: 449-459, 2005.

18. Duester G: Families of retinoid dehydrogenases regulating vitamin A function: Production of visual pigment and retinoic acid. Eur J Biochem 267: 4315-4324, 2000.

19. Kirwin SJ, Kanaly ST, Hansen CR, Cairns BJ, Ren M and Edelman JL: Retinal gene expression and visually evoked behavior in diabetic long evans rats. Invest Ophthalmol Vis Sci 52: 7654-7663, 2011.

20. Bullard JH, Purdom E, Hansen KD and Dudoit S: Evaluation of statistical methods for normalization and differential expression in mRNA-Seq experiments. BMC Bioinformatics 11: 94, 2010.

21. Seyednasrollah F, Laiho A and Elo LL: Comparison of software packages for detecting differential expression in RNA-seq studies. Brief Bioinform 16: 59-70, 2015.

22. Sheng WH, Sheng KT, Zhao YX, Li H, Zhou JL, Yao HY and Li XH: Identifying the biomarkers of multiple sclerosis based on non-coding RNA signature. Eur Rev Med Pharmacol Sci 19: 3635-3642, 2015

23. Hulsen T, de Vlieg $\mathbf{J}$ and Alkema W: BioVenn-a web application for the comparison and visualization of biological lists using area-proportional Venn diagrams. BMC Genomics 9: 488, 2008.

24. Vyas S and Kumaranayake L: Constructing socio-economic status indices: How to use principal components analysis. Health Policy Plan 21: 459-468, 2006.

25. Hild M, Beckmann B, Haas SA, Koch B, Solovyev V, Busold C, Fellenberg K, Boutros M, Vingron M, Sauer F, et al: An integrated gene annotation and transcriptional profiling approach towards the full gene content of the Drosophila genome. Genome Biol 5: R3, 2003.

26. Stafford R, Goodenough AE, Slater K, Carpenter W, Collins L, Cruickshank H, Downing S, Hall S, McDonald K, McDonnell $\mathrm{H}$, et al: Inferential and visual analysis of ethogram data using multivariate techniques. Animal Behaviour 83: 563-569, 2012.

27. Venables W and Ripely B: Modern Applied Statistics with S. 4th edition. Springer, New York, NY, 2002. 
28. Mao X, Zhang Y and Xu Y: SEAS: A system for SEED-based pathway enrichment analysis. PLoS One 6: e22556, 2011.

29. Xie C, Mao X, Huang J, Ding Y, Wu J, Dong S, Kong L, Gao G, Li CY and Wei L: KOBAS 2.0: A web server for annotation and identification of enriched pathways and diseases. Nucleic Acids Res 39 (Web Server Issue): W316-W322, 2011.

30. Agner F: Calculation Methods for Wallenius' Noncentral Hypergeometric Distribution. Commun Stat 37: 258-273, 2008.

31. Giot L, Bader J, Brouwer C, Chaudhuri A, Kuang B, Li Y, Hao YL, Ooi CE, Godwin B, Vitols E, et al: A protein interaction map of Drosophila melanogaster. Science 302: 1727-1736, 2003.

32. Li S, Armstrong CM, Bertin N, Ge H, Milstein S, Boxem M, Vidalain PO, Han JD, Chesneau A, Hao T, et al: A map of the interactome network of the metazoan C. elegans. Science 303: 540-543, 2004

33. Szklarczyk D, Franceschini A, Kuhn M, Simonovic M, Roth A, Minguez P, Doerks T, Stark M, Muller J, Bork P, et al: The STRING database in 2011: Functional interaction networks of proteins, globally integrated and scored. Nucleic Acids Res 39 (Database issue): D561-D568, 2011.

34. Smoot ME, Ono K, Ruscheinski J, Wang PL and Ideker T: Cytoscape 2.8: New features for data integration and network visualization. Bioinformatics 27: 431-432, 2011.

35. Yuan GS, Gao J, Zhang ZM, Du J, Mu GQ and Pan GT: Gene-Ontology analysis on the differentially expressed genes in maize (Zea mays L.) Ear Rot. J Life Sci 7: 219-226, 2013.

36. Huang da W, Sherman BT and Lempicki RA: Systematic and integrative analysis of large gene lists using DAVID bioinformatics resources. Nat Protoc 4: 44-57, 2009.

37. Zordoky BN, Aboutabl ME and El-Kadi AO: Modulation of cytochrome P450 gene expression and arachidonic acid metabolism during isoproterenol-induced cardiac hypertrophy in rats. Drug Metab Dispos 36: 2277-2286, 2008.

38. Chen W, Jump DB, Grant MB, Esselman WJ and Busik JV: Dyslipidemia, but not hyperglycemia, induces inflammatory adhesion molecules in human retinal vascular endothelial cells Invest Ophthalmol Vis Sci 44: 5016-5022, 2003

39. Joussen AM, Huang S, Poulaki V, Camphausen K, Beecken WD, Kirchhof B and Adamis AP: In vivo retinal gene expression in early diabetes. Invest Ophthalmol Vis Sci 42: 3047-3057, 2001.
40. Jensenius JC and Thiel S: MASP 2, a complement-fixing enzyme and uses for it. US Patent 20020082208 A1. Filed June 4, 2001; issued June 27, 2002.

41. Schwaeble HW, Stover CM, Tedford CE, Parent JB and Fujita T: Methods for treating conditions associated with MASP-2 dependent complement activation. WO Patent 2005123128 A2. Filed June 9, 2005; issued December 29, 2005.

42. Østergaard J, Hansen TK, Thiel S and Flyvbjerg A: Complement activation and diabetic vascular complications. Clin Chim Acta 361: 10-19, 2005.

43. Turner MW: The role of mannose-binding lectin in health and disease. Mol Immunol 40: 423-429, 2003.

44. Janecke AR, Thompson DA, Utermann G, Becker C, Hübner CA Schmid E, McHenry CL, Nair AR, Rüschendorf F, Heckenlively J, et al: Mutations in RDH12 encoding a photoreceptor cell retinol dehydrogenase cause childhood-onset severe retinal dystrophy. Nat Genet 36: 850-854, 2004.

45. Moiseyev G, Chen Y, Takahashi Y, Wu BX and Ma JX: RPE65 is the isomerohydrolase in the retinoid visual cycle. Proc Natl Acad Sci USA 102: 12413-12418, 2005.

46. Campochiaro PA: Seeing the light: New insights into the molecular pathogenesis of retinal diseases. J Cell Physiol 213: 348-354, 2007.

47. Huang P, Han J and Hui L: MAPK signaling in inflammation-associated cancer development. Protein Cell 1: 218-226, 2010.

48. Lawrence MC, Jivan A, Shao C, Duan L, Goad D, Zaganjor E, Osborne J, McGlynn K, Stippec S, Earnest S, et al: The roles of MAPKs in disease. Cell Res 18: 436-442, 2008.

49. Cuenda A and Nebreda AR: p38delta and PKD1: Kinase switches for insulin secretion. Cell 136: 209-210, 2009.

50. Sumara G, Formentini I, Collins S, Sumara I, Windak R, Bodenmiller B, Ramracheya R, Caille D, Jiang H, Platt KA, et al: Regulation of PKD by the MAPK p38delta in insulin secretion and glucose homeostasis. Cell 136: 235-248, 2009.

51. Osman N, Ballinger ML, Dadlani HM, Getachew R, Burch ML and Little PJ: p38 MAP kinase mediated proteoglycan synthesis as a target for the prevention of atherosclerosis. Cardiovasc Hematol Disord Drug Targets 8: 287-292, 2008. 